\title{
ON CLOSED GRAPH AND IMPLICIT FUNCTION THEOREMS FOR MULTIFUNCTIONS
}

\author{
C. C. CHOU, L. R. HUANG and K. F. NG
}

(Received 19 August 1994)

Communicated by E. N. Dancer

\begin{abstract}
We give several general implicit function and closed graph theorems for set-valued functions. Let $Z$ be a normed space, $X, Y$ metric spaces with $X$ complete. Let $f: X \rightrightarrows Z, F: X \times Y \rightrightarrows Z$ be multifunctions with $z_{0} \in f\left(x_{0}\right) \cap F\left(x_{0}, y_{0}\right)$ such that $f$ is open at $\left(x_{0}, y_{0}\right)$ and $f$ 'approximates' $F$ in an appropriate sense. Suppose that $f^{-1}(z)$ is closed, $F(x, y)$ is compact for each $x, y$ and $z$ and suppose that $F\left(x_{0}, \cdot\right)$ is lower semi-continuous at $y_{0}$. Then $F(\cdot, y)$ is of closed graph 'locally', is open at $x_{0}$, and there exists a function $x(\cdot)$ with $x(y) \rightarrow x_{0}$ for $y \rightarrow y_{0}$ such that $z_{0} \in F(x(y),(y))$ for all $y$ near $y_{0}$. A more general form dealing with the non-linear rate situation is also established.
\end{abstract}

1991 Mathematics subject classification (Amer. Math. Soc.): primary 46A30; secondary 58C15. Keywords and phrases: Multifunction, implicit function, open mapping theorem, implicit function theorem.

\section{Introduction}

Because of its importance in analysis as well as in applications, the Banach open mapping theorem has been studied and generalized by many authors in various aspects ever since its inception of functional analysis. Liusternik (cf. [11, 12]) gave open mapping and implicit function theorems for $C^{1}$ - functions; versions for some special classes of multifunctions such as convex processes began to appear in the mid-seventies $([13,18,19,22])$ as well as their latter extensions, for example, in [1-6, $9,10,14,16,17,20,23,24]$ and more recently in [21].

In this paper, we study multifunctions $f: X \rightrightarrows Z, F: X \rightrightarrows Z$ or, in the latter part, $F: X \times Y \rightrightarrows Z$, where $X, Y$ are metric spaces and $Z$ is a normed vector space. Let $\alpha, \delta$ be functions from $(0, \infty)$ into itself with $\delta$ increasing and $\lim _{t \rightarrow 0} \alpha(t)=0$. We define a type of open mapping property (called $(\delta, t)$-open in Definition 3.1 ) for $f$ relating to

(C) 1996 Australian Mathematical Society 0263-6115/96 $\$$ A2.00+0.00 
the validity of $B[z, \delta(t)] \subseteq f(B[x, t])$ for balls where $(x, z) \in f$. Since $\delta$ in general is non-linear, this new open mapping property is more general than the cases considered by many researchers whose conditions entail the Lipschitz requirements of the inverse $f^{-1}$. Nevertheless we show in Section 3 that our weaker assumptions still enable us to have interesting results in connection with the closed graph theorems about $f$ itself or $F$ provided that $F$ can be ' $\alpha$-approximated' by $f$. The $\alpha$-approximation is defined in Definition 3.2. If $f, F$ are single-valued and $\alpha(t)=l t$ for constant $l \in(0, \infty)$, this property says nothing but $f-F$ is a Lipschitz function with modulus $l$. Suppose that $\alpha \prec \delta$ in the sense that there exists a decreasing sequence $\left(t_{n}\right)$ such that $\alpha\left(t_{n}\right) \leq \delta\left(t_{n+1}\right)$ for all $n$ (for example this is the case if $\alpha=(l t)^{p}, \delta=(c t)^{p}$ with $0<l<c$ and $p>0$, or $\alpha=(l t)^{q}, \delta=(c t)^{p}$ with $q>p>0$ and $\left.l, c>0\right)$. We establish in Section 4 that $F$ is an open mapping when $f$ is $(\delta(t), t)$-open and $\alpha$-approximates $F$ under some reasonable conditions (such as $F$ is of compact-values). This result is then applied to prove a general implicit function theorem (Theorem 4.3) dealing with, in general, non-linear and not-one-one cases. Specializing to the case when $f, F$ are single-valued, $f$ is $1-1$ and both $\alpha, \delta$ are linear then our implicit function result is a mild extension of a recent result of Robinson [21].

Our method is based on the open mapping theorem recently obtained by Chou [6] while the main tool in [21] is a fixed point principle. In Theorem 2.1 we give a version of this theorem applicable even to functions whose graphs are not necessarily closed. This new version is needed in our study in Section 4 for implicit function theorems. Section 3 is devoted to some results on closed graphs of multifunctions.

For simplicity of notation we use the same letter $d$ to denote the given metrics for $X$ and $Y$; and likewise for $\rho \geq 0, B[a, \rho]$ denotes the closed ball (with center $a$ and radius $\rho$ ) in $X, Y$ or $Z$ - depending on $a$ being an element of $X, Y$ or $Z$. If $A$ is a set, then $B[A, \rho]$ is the union of all $B[a, \rho]$ as $a$ runs over all of $A$. We sometimes write $A_{\rho}$ for the set $B[A, \rho]$ and $A^{\rho}:=\{x: B[x, \rho] \subseteq A\}$. A multifunction $f: X \rightrightarrows Z$ is identified with its graph

$$
f:=\{(x, z) \in X \times Z: z \in f(x)\} .
$$

$\operatorname{Dom}(f)$ denotes the set $\{x \in X: \exists z$ such that $z \in f(x)\}$.

We denote by $f^{-1} \subseteq Z \times X$ the multifunction given by

$$
f^{-1}:=\{(z, x) \in Z \times X:(x, z) \in f\} .
$$

For a subset $A$ of $X$, we denote by $f(A)$ the set $\bigcup_{x \in A} f(x)$.

\section{Controlled triples and open mappings}

Let $\left(\delta_{n}\right),\left(t_{n}\right)$ and $\left(\alpha_{n}\right)$ be sequences of positive real numbers such that $\delta_{n+1} \leq \delta_{n}$ for each $n$ and $\lim _{n \rightarrow \infty} \alpha_{n}=0$. We call $\left(\delta_{n}, t_{n}, \alpha_{n}\right)$ a controlled triple if $0<\alpha_{n} \leq$ 
$\delta_{n+1} \leq \delta_{n}$ for each $n$ and $T_{n}:=\sum_{j=n}^{\infty} t_{j}<+\infty$.

Part (ii) of the following lemma is easy to verify while (i) follows immediately from (ii).

LEMMA 2.1. (i) Let $0<\tau ; 0<l<c$ and $q=l / c$. Then $\left(\delta_{n}, t_{n}, \alpha_{n}\right)$ is a controlled triple where $t_{n}:=\tau q^{n}, \delta_{n}:=c t_{n}$ and $\alpha_{n}:=l t_{n}$.

(ii) Let $\delta, \alpha$ be functions on $(0, \infty)$ into itself with $\delta$ increasing and $\lim _{t \rightarrow 0} \alpha(t)=$ 0 . Let $\left(t_{n}\right)$ be a decreasing sequence of positive real numbers such that $\sum_{i=1}^{\infty} t_{i}<+\infty$ and $\alpha\left(t_{n}\right) \leq \delta\left(t_{n+1}\right)$ for each $n$. Then $\left(\delta\left(t_{n}\right), t_{n}, \alpha\left(t_{n}\right)\right)$ is a controlled triple.

We shall use the notation $\alpha \prec \delta$ if $\alpha, \delta$ satisfy the conditions of Lemma 2.1 (ii).

EXAMPLE 2.1. Let $0<l<c$ and $p>0$. Let $\alpha(t)=(l t)^{p}$ and $\delta(t)=(c t)^{p}$. Then for $t_{n}=(l / c)^{n}$, one has $\alpha\left(t_{n}\right) \leq \delta\left(t_{n+1}\right)$ for all $n$. Consequently $\alpha \prec \delta$.

EXAMPLE 2.2. Let $0<l, c$ and $q>p>0$. Let $\alpha(t)=(l t)^{q}$ and $\delta(t)=(c t)^{p}$. Then for $t_{n}=k^{n}$ with any positive constant $k<1$, one has $\alpha\left(t_{n}\right) \leq \delta\left(t_{n+1}\right)$ for all large $n$. Consequently $\alpha \prec \delta$.

The following result slightly extends that in [3] and [6], and encompasses many 'auto-open' mapping results, for example [5, 10, 15 and 23]; see [6] for details.

Let $E, G$ be metric spaces, and $N, g, F \subseteq E \times G$ such that $\bar{g} \subseteq F$.

THEOREM 2.1. Let $s>0$,

$$
N_{s}:=\{(x, y) \in E \times G: \exists(a, b) \in N \text { such that } d(x, a) \leq s, d(y, b) \leq s\}
$$

and $\left(\delta_{n}, t_{n}, \alpha_{n}\right)$ be a controlled triple such that

$$
B\left[y, \delta_{j}\right] \subseteq B\left[g\left(B\left[x, t_{j}\right]\right), \alpha_{j}\right] \quad \forall j
$$

whenever $(x, y) \in g \cap N_{s}$. Suppose that $\operatorname{Dom}(g)$ is pre-complete in $E$ in the sense that any Cauchy sequence $\left(x_{n}\right)$ in $\operatorname{Dom}(g)$ has a limit in $E$. If $s \geq 2 \delta_{i}, T_{i}$, then

$$
B\left[y, \delta_{i}\right] \subseteq F\left(B\left[x, T_{i}\right]\right) \quad \text { whenever }(x, y) \in g \cap N .
$$

PROOF. Let $(x, y) \in g \cap N$, and $z \in B\left[y, \delta_{i}\right]$. Then there exist $x_{1} \in B\left[x, t_{i}\right]$ and $y_{1} \in g\left(x_{1}\right)$ such that $d\left(z, y_{1}\right) \leq \alpha_{i}\left(\leq \delta_{i+1}\right)$. Then $\left(x_{1}, y_{1}\right) \in g \cap N_{s}$ so there exist $x_{2} \in B\left[x_{1}, t_{i+1}\right]$ and $y_{2} \in g\left(x_{2}\right)$ such that $d\left(z, y_{2}\right) \leq \alpha_{i+1}\left(\leq \delta_{i+2}\right)$. Inductively, we have a Cauchy sequence $\left(x_{n}\right)$ in $\operatorname{Dom}(g)$, say with limit $\bar{x}$ such that $g\left(x_{n}\right) \ni y_{n} \rightarrow z$. By the assumption that $\bar{g} \subseteq F$, we have $(\bar{x}, z) \in F$. Clearly $\bar{x} \in B\left[x, T_{i}\right]$ so $z \in F\left(B\left[x, T_{i}\right]\right)$. 
REMARK. Suppose $g$ is of closed graph, and consider $i$ large enough such that

$$
s \geq 4 \delta_{i}, 2 T_{i} .
$$

Let $Y$ denote the set consisting of all $y$ for each of which there exists $x$ such that $(x, y) \in g \cap N$. Let $W=Y+B\left[0, \delta_{i}\right]$. By (2.1) we know that $W$ is contained in the range of $g$ so $g^{-1}(w)$ is non-empty for each $w$ in $W$. In this situation we have the following 'continuity property' of the inverse map $g^{-1}$ in terms of the distance defined by

$$
\operatorname{dist}(A, B)=\inf \{d(a, b): a \in A, b \in B\} .
$$

COROLlaRY 1 . Let $j \geq i$. Then $\operatorname{dist}\left(g^{-1}\left(w_{1}\right), g^{-1}\left(w_{2}\right)\right) \leq T_{j}$ whenever $w_{1}, w_{2} \in$ $W$ and $d\left(w_{1}, w_{2}\right) \leq \delta_{j}$.

PROOF. Let $w_{1}, w_{2} \in W$ with $d\left(w_{1}, w_{2}\right) \leq \delta_{j}$, where $j \geq i$. By definition of $W$ and $Y$, pick $y_{1} \in Y$ and then $x \in E$ such that $d\left(w_{1}, y_{1}\right) \leq \delta_{i}$ and $\left(x, y_{1}\right) \in g \cap N$. It follows from (2.1) that

$$
w_{1} \in B\left[y_{1}, \delta_{i}\right] \subseteq g\left(B\left[x, T_{i}\right]\right)
$$

so $w_{1} \in g\left(x_{1}\right)$ for some $x_{1} \in B\left[x, T_{i}\right]$. By (2.2) it follows that $\left(x_{1}, w_{1}\right) \in g \cap N_{s / 2}$. In view of (2.2) again one may apply Theorem 2.1 with $s / 2, N_{s / 2}$ in place of $s, N$; thus

$$
w_{2} \in B\left[w_{1}, \delta_{j}\right] \subseteq g\left(B\left[x_{1}, T_{j}\right]\right)
$$

and so $w_{2} \in g\left(x_{2}\right)$ for some $x_{2} \in B\left[x_{1}, T_{j}\right]$. Therefore

$$
\operatorname{dist}\left(g^{-1}\left(w_{1}\right), g^{-1}\left(w_{2}\right)\right) \leq d\left(x_{1}, x_{2}\right) \leq T_{j} .
$$

\section{Closed Graph Theorems}

Throughout we use $\alpha, \delta$ to denote functions on $(0, \infty)$ into itself and we suppose that $\delta$ is increasing and $\alpha(t) \rightarrow 0$ as $t \rightarrow 0$. Let $U \subseteq X$ and $V \subseteq Z$.

DEFINITION 3.1. A multifunction $f: X \rightrightarrows Z$ is said to be $(\delta(t), t)$-open (uniformly) with respect to $(U, V)$ if there exists $\tau>0$ such that, for each $(x, z) \in$ $f \cap(U \times V)$,

$$
B[z, \delta(t)] \subseteq f(B[x, t]), \quad \forall t \in(0, \tau]
$$

(in this case, we also say that $f$ is $(\delta(t), t)$-open with respect to $(U, V, \tau))$. If $U=X, V=Z$ then we simply say $f$ is $(\delta(t), t)$-open. 
REMARK 1 . The above property is hereditary when $U \times V$ is replaced by its subset.

REMARK 2. The condition (3.1) of course ensures that $f$ is open at $x$ in the usual sense.

REMARK 3. Let $\left(\delta_{n}, t_{n}, \alpha_{n}\right)$ be a controlled triple satisfying the conditions of Theorem 2.1. Take large enough $i$ such that $s \geq 2 \delta_{i}, T_{i}$. For each $j \geq i$, define $\gamma\left(T_{j}\right)=\delta_{j+1}$, and then extend to define a strictly increasing function $\gamma: \mathbb{R}^{+} \rightarrow \mathbb{R}^{+}$ whose graph contains the union of the line-segments joining the consecutive points $\left(T_{j}, \gamma\left(T_{j}\right)\right)$. Note that if $t \in\left(T_{j+1}, T_{j}\right]$ then $\gamma(t) \leq \delta_{j+1}$ and it follows from (2.1) that

$$
B[y, \gamma(t)] \subseteq B\left[y, \delta_{j+1}\right] \subseteq F\left(B\left[x, T_{j+1}\right]\right) \subseteq F(B[x, t])
$$

for all $(x, y) \in g \cap N$. Thus, with the obvious adoption of Definition 3.1 (for $g \cap N$ instead of $U \times V), F$ is $(\gamma(t), t)$-open with respect to $g \cap N$.

REMARK 4. In [21], Robinson considered the following: Let $f$ be a single-valued function with $f: \sum \rightarrow Z, f(\Sigma) \supseteq B\left[z_{0}, R\right]$ for some $R>0$, and he supposed that

$$
d_{0}:=\inf \left\{\frac{\left|f(x)-f\left(x^{\prime}\right)\right|}{d\left(x, x^{\prime}\right)}: x, x^{\prime} \in \Sigma, x \neq x^{\prime}\right\}>0 .
$$

If we let $r \in(0, R), B_{r}:=B\left[z_{0}, r\right]$ and $\tau=(R-r) d_{0}^{-1}$, then $f$ is $\left(d_{0} t, t\right)$ open with respect to $\left(\Sigma, B_{r}, \tau\right)$. In fact let $\bar{z} \in B\left[z, d_{0} t\right]$ where $t \in(0, \tau]$ and $(x, z) \in f \cap\left(\Sigma \times B_{r}\right)$. Then $\left|\bar{z}-z_{0}\right| \leq d_{0} t+r \leq R$ and so $\bar{z}=f(\bar{x})$ for some $\bar{x} \in \Sigma$. Further, by definition of $d_{0}$,

$$
d_{0} d(x, \bar{x}) \leq|z-\bar{z}| \leq d_{0} t
$$

proving that $\bar{x} \in B[x, t]$ and hence that $B\left[z, d_{0} t\right] \subseteq f(B[x, t])$. The same remark and its proof are valid even for a multifunction $f: \Sigma \rightrightarrows Z$ with $d_{0}$ to be defined by

$$
d_{0}:=\inf \left\{\frac{\left|z-z^{\prime}\right|}{d\left(x, x^{\prime}\right)}: x, x^{\prime} \in \Sigma, x \neq x^{\prime}, z \in f(x), z^{\prime} \in f\left(x^{\prime}\right)\right\}
$$

Note that if $d_{0}>0$ then $f(x)$ and $f\left(x^{\prime}\right)$ must be disjoint whenever $x \neq x^{\prime}$. Thus, for single-valued functions, the condition $d_{0}>0$ implies that $f$ is 1-1. The converse is not true, for example, $f: t \longrightarrow t^{3}$ is open and 1-1 but the corresponding $d_{0}=0$. Thus this example is not covered by results in [21], but satisfies the following lemma with $\delta: t \longrightarrow t^{3}$. 
LEMMA 3.1. Let $\delta: \mathbb{R}^{+} \longrightarrow \mathbb{R}^{+}$be a strictly increasing function and $f: X \rightrightarrows Z$ with $f(\Sigma) \supseteq B\left[z_{0}, R\right]$ where $\Sigma \subseteq X, z_{0} \in Z$ and $R>0$. Define

$$
\delta(f, \Sigma):=\inf \left\{\frac{\left|z_{1}-z_{2}\right|}{\delta\left(d\left(x_{1}, x_{2}\right)\right)}: \text { each } x_{i} \in \Sigma, z_{i} \in f\left(x_{i}\right) \text { and } x_{1} \neq x_{2}\right\} .
$$

Suppose $c:=\delta(f, \Sigma)>0$. Then $f$ is $(c \delta(t), t)$-open with respect to $\left(\Sigma, B_{r}, \tau\right)$ where $r \in(0, R), B_{r}:=B\left[z_{0}, r\right]$ and $\tau=\delta^{-1} c^{-1}(R-r)$.

EXAMPLE 3.1. Let $p \geq 1$. Let $f(t)=(\operatorname{sgn} t)|t|^{p}$ for all $t \in \mathbb{R}$, and $\delta$ the restriction of $f$ to $\mathbb{R}^{+}$. Let $X=Z=\Sigma=\mathbb{R}$. Then $\delta(f, \Sigma)=(1 / 2)^{p-1}$. Indeed, by convexity, $[(x+t) / 2]^{p} \leq\left(x^{p}+t^{p}\right) / 2, \forall x, t \geq 0$ and it follows that

$$
\inf \left\{\frac{x^{p}+t^{p}}{(x+t)^{p}}: x, t>0\right\}=\frac{1}{2^{p-1}} .
$$

Also, since $t^{p}+(1-t)^{p} \leq 1, \forall t \in(0,1)$, it follows that

$$
\inf \left\{\frac{x^{p}-t^{p}}{(x-t)^{p}}: x>t>0\right\}=1 .
$$

Consequently

$$
\begin{aligned}
\delta(f, \Sigma) & =\inf \left\{\frac{x^{p}-t^{p}}{(x-t)^{p}}: x>t>0\right\} \wedge \inf \left\{\frac{x^{p}+t^{p}}{(x+t)^{p}}: x, t>0\right\} \\
& =\min \left\{1, \frac{1}{2^{p-1}}\right\}=\frac{1}{2^{p-1}} .
\end{aligned}
$$

THEOREM 3.1. Suppose $f: X \rightrightarrows Z$ is $(\delta(t), t)$-open. Then $f$ is of closed graph if and only if $f^{-1}(z)$ is closed for all $z \in Z$.

The necessity part is easy to verify while the sufficiency part follows from the following theorem (applied to $U=X$ and $V=Z$ ).

THEOREM 3.2. Suppose $f: X \rightrightarrows Z$ is $(\delta(t), t)$-open with respect to $U \times V$, and that $f^{-1}(z)$ is closed for all $z \in \bar{V}$. Then the closure of the restriction $\left.f\right|_{U \times V}$ of $f$ is contained in $f$.

PROOF. Let $(\bar{x}, \bar{z})$ be the limit of a sequence $\left(x_{n}, z_{n}\right) \in f \cap(U \times V)$. Then $\bar{z} \in \bar{V}$. Take $\tau>0$ such that (3.1) in Definition 3.1 holds. Consider $t_{k}$ of the form $t_{k}=1 / k$ with positive integers $k$, large enough so that $t_{k}<\tau$. Take a strictly increasing sequence $n_{k}$ of positive integers such that $d\left(z_{n_{k}}, \bar{z}\right)<\delta\left(t_{k}\right)$. Then it follows from (3.1) that

$$
\bar{z} \in B\left[z_{n_{k}}, \delta\left(t_{k}\right)\right] \subseteq f\left(B\left[x_{n_{k}}, t_{k}\right]\right)
$$


and so $\bar{z} \in f\left(\xi_{k}\right)$ for some $\xi_{k} \in B\left[x_{n_{k}}, t_{k}\right]$. Passing to the limit in $\xi_{k} \in f^{-1}(\bar{z})$, we have $\bar{x} \in f^{-1}(\bar{z})$, that is, $(\bar{x}, \bar{z}) \in f$.

REMARK. There are simple examples of $(\delta(t), t)$-open mappings with non-closed graphs.

Let, as before, $f: X \rightrightarrows Z, F: X \rightrightarrows Z$. Let $U \subseteq X$, and $V, V^{f} \subseteq Z$. Recall that $\alpha: \mathbb{R}^{+} \longrightarrow \mathbb{R}^{+}$with $\alpha(t) \rightarrow 0$ when $t \rightarrow 0$.

DEFINITION 3.2. $f$ is said to $\alpha$-approximate (innerly) $F$ with respect to $\left(U, V, V^{f}\right)$ if there exists $\tau>0$ such that for each $x \in U$ and $z^{F} \in F(x) \cap V$ there exists $z^{f} \in f(x) \cap V^{f}$ with the property

$$
f\left(x^{\prime}\right)-z^{f} \subseteq B\left[F\left(x^{\prime}\right)-z^{F} ; \alpha(t)\right], \quad \forall d\left(x^{\prime}, x\right) \leq t \leq \tau .
$$

If $V=V^{f}$ then we simply say that $f \alpha$-approximates $F$ with respect to $(U, V)$.

REMARK 1. This property is hereditary if $U$ is replaced by its subset.

REMARK 2. If $f, F$ are single-valued then $(*)$ can be rewritten as

$$
\left|f\left(x^{\prime}\right)-F\left(x^{\prime}\right)-[f(x)-F(x)]\right| \leq \alpha(t), \quad \forall d\left(x^{\prime}, x\right) \leq t \leq \tau .
$$

So if $V=F(U), V^{f}=f(U)$ and $\alpha(t)$ is linear: $\alpha(t)=l t, \forall t$ with some constant $l$ then this simply is equivalent to: $f-F$ is Lipschitz (with modulus $l$ on the $\tau$ neighbourhood of $U$ ).

REMARK 3. For any $f: X \rightrightarrows Z$ and $K \subseteq Z$, let

$$
F(x)=f(x)+K, \quad \forall x \in X
$$

Then $f \alpha$-approximates $F$ with respect to $(U, V, V-K)$ for any $\alpha$ and $U \times V \subset X \times Z$.

The following theorem is clearly a special case of Theorem 3.4 .

THEOREM 3.3. Suppose that $\overline{\operatorname{Dom}(F)} \subseteq \operatorname{Dom}(f)$ and

(i) $f$ is of closed graph,

(ii) $f \alpha$-approximates $F$ (that is, with respect to $(X, Z)$ ),

(iii) $F(x)$ is compact for all $x \in X$.

Then $F$ is of closed graph.

THEOREM 3.4. Let $U \subseteq X$ and $V, V^{f} \subseteq Z$. Suppose that $\overline{\operatorname{Dom}(F)} \subseteq \operatorname{Dom}(f)$ and 
(i) The closure of the restriction of $f$ to $U \times V^{f}$ is contained in $f$,

(ii) $f \alpha$-approximates $F$ with respect to $\left(U, V, V^{f}\right)$,

(iii) $F(x)$ is compact for all $x \in \bar{U}$.

Then the closure of the restriction $\left.F\right|_{U \times V}$ of $F$ to $U \times V$ is contained in $F$. Consequently $\left.F\right|_{U \times V}$ is closed provided that $U$ and $V$ are closed.

PROOF. By (ii), take $\tau>0$ so that the situation in Definition 3.2 holds. Let $(\bar{x}, \bar{z})$ be the limit of a sequence $\left(x_{n}, z_{n}^{F}\right) \in F \cap(U \times V)$. Then $x_{n}, \bar{x} \in \operatorname{Dom}(f)$, and moreover we may assume that $d\left(\bar{x}, x_{n}\right)<\tau$ for each $n$, hence there exists $z_{n}^{f} \in f\left(x_{n}\right) \cap V^{f}$ such that

$$
f(\bar{x})-z_{n}^{f} \subseteq B\left[F(\bar{x})-z_{n}^{F} ; \alpha\left(d\left(\bar{x}, x_{n}\right)\right)\right] .
$$

Pick $z \in f(\bar{x})$, and then take $\xi_{n} \in F(\bar{x})$ such that

$$
\left|z-z_{n}^{f}-\left(\xi_{n}-z_{n}^{F}\right)\right| \leq \alpha\left(d\left(\bar{x}, x_{n}\right)\right) .
$$

Since $F(\bar{x})$ is compact by (iii), we may suppose that $\xi_{n} \rightarrow \xi$ for some $\xi \in F(\bar{x})$. It follows by passing to the limit in (3.4) that $z_{n}^{f} \rightarrow z-\xi+\bar{z}$. Thus $(\bar{x}, z-\xi+\bar{z})$ is the limit of $\left(x_{n}, z_{n}^{f}\right) \in f \cap\left(U \times V^{f}\right)$ and it follows from (i) that $z-\xi+\bar{z} \in f(\bar{x})$. Thus, by (3.3), (3.4) continues to hold if $z$ is replaced by $z-\xi+\bar{z}$. Comparing this new inequality with (3.4) we see that $-\xi+\bar{z}=0$; hence $\bar{z}=\xi \in F(\bar{x})$.

THEOREM 3.4* Let $W \subseteq X, V \subseteq Z, V^{f} \subseteq Z$ be closed sets, and $U=W \cap$ $f^{-1}\left(V^{f}\right)$. Then $\left.F\right|_{U \times V}$ is closed if (i), (ii) and (iii) of Theorem 3.4 are satisfied, and $\overline{\operatorname{Dom}(F)} \subseteq \operatorname{Dom}(f)$.

PROOF. The same proof works: if $(\bar{x}, \bar{z}) \in \overline{F \cap(U \times V)}$ then $\bar{x} \in \bar{U} \subseteq W$; also $z-\xi+\bar{z}=\lim _{n \rightarrow \infty} z_{n}^{f} \in \overline{V^{f}}=V^{f}$ and it follows from $z-\xi+\bar{z} \in f(\bar{x})$ that $\bar{x} \in f^{-1}\left(V^{f}\right)$. Therefore $\bar{x} \in U$ and consequently $(\bar{x}, \bar{z}) \in F \cap(U \times V)$

REMARK. $U$ is not necessarily closed in Theorem $3.4^{*}$, as $f$ is not assumed continuous.

Combining Theorems $3.2,3.4$ and $3.4^{*}$, we have

THEOREM 3.5. Suppose that $\overline{\operatorname{Dom}(F)} \subseteq \operatorname{Dom}(f)$ and

(i) $f$ is $(\delta(t), t)$-open with respect to $\left(U, V^{f}\right)$;

(ii) $f$ a-approximates $F$ with respect to $\left(U, V, V^{f}\right)$;

(iii) $f^{-1}(z)$ is closed for each $z \in \overline{V^{f}}$;

(iv) $F(x)$ is compact for each $x \in \bar{U}$.

Suppose further that either (a) $U, V$ are also closed or (b) $V$ is closed and $U=$ $f^{-1}\left(V^{f}\right) \cap W$ for some closed sets $W$ and $V^{f}$. Then $\left.F\right|_{U \times V}$ is of closed graph. 


\section{Open mapping and implicit functions}

Let $U, V, V^{f}, \alpha, \delta$ be as at the beginning of the preceeding section. We suppose further that $U$ is precomplete in $X$. Let $f: X \rightrightarrows Z, F: X \rightrightarrows Z$. We suppose throughout this section that $\overline{\operatorname{Dom}(F)} \subseteq \operatorname{Dom}(F)$. To prepare the main results, we begin with:

\section{THEOREM 4.1. Suppose that}

(i) $f$ is $(\delta(t), t)$-open with respect to $\left(U, V^{f}\right)$;

(ii) $f \alpha$-approximates $F$ with respect to $\left(U, V, V^{f}\right)$.

Then we have

$$
B[z, \delta(t)] \subseteq B[F(B[x, t]) ; \alpha(t)], \quad \forall t \in(0, \tau]
$$

whenever $x \in U$ and $z \in F(x) \cap V$.

Proof. By (i) and (ii), we may take the same $\tau>0$ in Definitions 3.1 and 3.2. Let $x \in U, z^{F} \in F(x) \cap V$ and $t \in(0, \tau]$. By (ii), there exists $z^{f} \in V^{f} \cap f(x)$ such that

$$
f(B[x, t])-z^{f} \subseteq B\left[F(B[x, t])-z^{F} ; \alpha(t)\right], \quad \forall t \in(0, \tau]
$$

By (i), we also have, for this $\left(x, z^{f}\right)$ in $f \cap\left(U \times V^{f}\right)$, that

$$
B\left[z^{f}, \delta(t)\right] \subseteq f(B[x, t]), \quad \forall t \in(0, \tau] .
$$

Hence

$$
B[0, \delta(t)] \subseteq f(B[x, t])-z^{f} \subseteq B\left[F(B[x, t])-z^{F} ; \alpha(t)\right],
$$

that is,

$$
B\left[z^{F}, \delta(t)\right] \subseteq B[F(B[x, t]) ; \alpha(t)], \quad \forall t \in(0, \tau],
$$

proving (4.1).

For the remainder of this paper we assume that the function $\alpha, \delta$ satisfy an additional requirement $\alpha \prec \delta$, namely that there exists a decreasing sequence $\left(t_{n}\right)$ of positive real numbers such that $\sum t_{n}<+\infty$ and $\alpha\left(t_{n}\right) \leq \delta\left(t_{n+1}\right)$ for each $n$. There are an abundance of examples of such $\alpha, \delta$, as explained in Section 2. Recall that, for any set $N$ in a metric space and $s>0, N^{s}$ denotes the set $\{x: B[x, s] \subseteq N\}$ and $N_{s}:=B[N, s]$. 
THEOREM 4.2. In additon to the assumptions (i), (ii) of Theorem 4.1 with $\alpha \prec \delta$, we suppose further that

(iii) $f^{-1}(z)$ is closed for each $z \in \overline{V^{f}}$,

(iv) $F(x)$ is compact for each $x \in \vec{U}$.

Let $s>0$. Then, for all $(x, z) \in F \cap\left(U^{3 s} \times V^{3 s}\right)$, one has

$$
B\left[z, \delta\left(t_{n}\right)\right] \subseteq F\left(B\left[x, T_{n}\right]\right)
$$

whenever $n$ is large enough that $\alpha\left(t_{n}\right), T_{n}, 2 \delta\left(t_{n}\right)<\min \{s, \tau\}$, where $T_{n}:=\sum_{j=n}^{\infty} t_{j}$.

ProOF. We suppose without loss of generality that $\tau<s$ and $t_{n} \in(0, \tau]$ for all $n$ (take smaller $\tau$ in (i), (ii) and delete finitely many terms of $\left(t_{n}\right)$, if necessary). By Theorem 4.1, for each $(x, z) \in(U \times V) \cap F$, one has

$$
B\left[z ; \delta\left(t_{n}\right)\right] \subseteq B\left[F\left(B\left[x, t_{n}\right]\right) ; \alpha\left(t_{n}\right)\right] \quad \forall n .
$$

Henceforth we consider $n$ large enough in the sense already specified. Let $g=\left.F\right|_{U \times V}$, and $N:=U^{3 s} \times V^{3 s}$. Note that if $(x, z) \in N_{s} \cap g$ then $(x, z) \in U^{2 s} \times V^{2 s}$ and satisfies (4.3). Consequently

$$
B\left[z ; \delta\left(t_{n}\right)\right] \subseteq B\left[g\left(B\left[x, t_{n}\right]\right) ; \alpha\left(t_{n}\right)\right]
$$

Indeed, if $\xi^{\prime}$ is from the left member of (4.3*) then, by (4.3), $\left|\xi-\xi^{\prime}\right| \leq \alpha\left(t_{n}\right)<s$ for some $\xi \in F(\eta)$ and some $\eta \in B\left[x ; t_{n}\right]$. Hence

$$
|z-\xi| \leq\left|z-\xi^{\prime}\right|+\left|\xi-\xi^{\prime}\right| \leq \delta\left(t_{n}\right)+s \leq 2 s .
$$

Therefore $\xi \in z+B[0,2 s] \subseteq V$ since $z \in V^{2 s}$. Similarly $\eta \in U$. This means that the relations $\xi \in F(\eta)$ and $\xi \in g(\eta)$ are the same, and so $\left(4.3^{*}\right)$ follows from (4.3). Now, since by Theorem 3.4 , the closure of $g$ is contained in $F$, we apply Theorem 2.1 to conclude that $(4.2)$ holds thanks to $\left(4.3^{*}\right)$.

Local Theory We shall consider multifunctions $F: X \times Y \rightrightarrows Z$ and $f: X \rightrightarrows Z$. For $y \in Y$ we use $F^{y}$ to denote the multifunction $F(\cdot, y)$. Let $\left(x_{0}, y_{0}\right) \in X \times Y$, and $z_{0} \in F\left(x_{0}, y_{0}\right)$. Let $U$ be a neighbourhood of $x_{0}$. We suppose further that $U$ is precomplete in $X$. Let $V$ be a neighbourhood of $z_{0}$. The following result shows the existence of an implicit function for $z_{0} \in F(x(\cdot), \cdot)$ near $y_{0}$ if $F\left(x_{0}, \cdot\right)$ is lower semi-continuous at $y_{0}$ and if $f, F^{y}$ satisfy the conditions of the preceeding theorem for each $y$ near $y_{0}$.

THEOREM 4.3. Let $\rho>0$, and $\alpha \prec \delta$. Suppose that

(i) $f$ is $(\delta(t), t)$-open with respect to $\left(U, V^{f}\right)$, 
(ii) $f \alpha$-approximates $F^{y}$ with respect to $\left(U, V, V^{f}\right)$ for each $y \in B\left[y_{0} ; \rho\right]$,

(iii) $f^{-1}(z)$ is closed for each $z \in \overline{V^{f}}$,

(iv) $F(x, y)$ is compact for each $x \in \bar{U}$ and $y \in B\left[y_{0}, \rho\right]$,

(v) $F\left(x_{0}, \cdot\right)$ is lower semi-continuous at $y_{0}$.

Then there exists a neighbourhood $M$ of $x_{0}$, a neighbourhood $P$ of $y_{0}$ and a singlevalued function $x(\cdot)$ on $P$ to $M$ such that $z_{0} \in F(x(y), y)$ for all $y \in P$ with $x(\cdot)$ continuous at $y_{0}$ and $x\left(y_{0}\right)=x_{0}$.

Granting this, we have the following:

COROLlARY 1. Let $l, c$ be constants with $0<l<c$, and $\alpha(t):=l t, \delta(t):=c t$ $\left(\right.$ so $\alpha \prec \delta$ with $\left.t_{n}:=(l / c)^{n}\right)$. Then Theorem 4.3 remains valid if (i), (ii) are replaced by

(i*) $f$ is $(c t, t)$-open with respect to $\left(U, V^{f}\right)$,

(ii*) $f \alpha$-approximates $F^{y}$ with respect to $\left(U, V, V^{f}\right)$ for each $y \in B\left[y_{0}, \rho\right]$.

In view of Lemma 3.1 (and replacing $X$ by $\Sigma$ in Theorem 4.3), we also have:

COROLLARY 2. Let $\alpha \prec \delta$ with strictly increasing $\delta$, and let $U$ be any precomplete neighbourhood of $x_{0}$ in the subspace $\Sigma$ of $X$ under the relative topology. Let $f$ : $X \rightrightarrows Z$, and suppose that $f(\Sigma) \supseteq B\left[z_{0}, R\right]$ for some $R>0$. Let $V \subseteq B\left[z_{0}, r\right]$ be a neighbourhood of $z_{0}$ with $r \in(0, R)$. Let (i), (ii) in Theorem 4.3 be replaced by

$$
c=\delta(f, \Sigma):=\inf \left\{\frac{\left|z-z^{\prime}\right|}{\delta\left(d\left(x, x^{\prime}\right)\right)}: x, x^{\prime} \in \Sigma, x \neq x^{\prime}, z \in f(x), z^{\prime} \in f\left(x^{\prime}\right)\right\}>0,
$$

(ii**) $f$ c $\alpha$-approximates $F^{y}$ with respect to $(U, V)$ for each $y \in B\left[y_{0}, \rho\right]$.

and other assumptions (that is, (iii)-(v) unchanged). Then the conclusions of Theorem 4.3 remain true.

(By Lemma 3.1 and Remark 1 after Definition 3.1, $\left(\mathrm{i}^{* *}\right)$ implied that $f$ is $(c \delta(t), t)$ open with respect to $U, V$.)

NOTE. In particular we have the following Corollary 3 which was established by Robinson [21] in the special case when $f, F$ are single-valued, $p=1, c=1, \alpha(t)=l t$ and under a stronger approximation property than $\left(\mathrm{ii}^{* *}\right)$.

COROLlaRY 3. Let $p>0, k>0$ and $\delta(t)=(k t)^{p}$. Let $\alpha$ be

either $\alpha(t)=(l t)^{p}, \quad$ where $l$ is a positive constant with $l<k$,

or $\alpha(t)=t^{q} \quad$ with $q>p$. 
Then $\alpha \prec \delta$ and hence Theorem 4.3 remains true if (i), (ii) are replaced by

$$
c:=\inf \left\{\frac{\left|z-z^{\prime}\right|}{k^{p} d\left(x, x^{\prime}\right)^{p}}: x, x^{\prime} \in \Sigma, x \neq x^{\prime}, z \in f(x), z^{\prime} \in f\left(x^{\prime}\right)\right\}>0,
$$

(ii ${ }^{\# \#) ~} f$ c $\alpha$-approximates $F^{y}$ with respect to $(U, V)$ for each $y \in B\left[y_{0}, \rho\right]$.

ProOF OF THEOREM 4.3. Take the same $\tau>0$ in Definitions 3.1 and 3.2. Take $s>0$ such that $B\left[x_{0}, 3 s\right] \subseteq U$; thus $x_{0} \in U^{3 s}$. We may further assume that $z_{0} \in V^{4 s}$. Take a positive integer $n_{0}$, large enough so that $\alpha\left(t_{n}\right), T_{n}, 2 \delta\left(t_{n}\right)<\min \{s, \tau\}$ for all $n \geq n_{0}$. Let $y \in B\left[y_{0}, \rho\right]$. By Theorem 4.2, we have

$$
B\left[F^{y}\left(x_{0}\right) \cap V^{3 s} ; \delta\left(t_{n}\right)\right] \subseteq F^{y}\left(B\left[x_{0}, T_{n}\right]\right), \quad \forall n \geq n_{0} .
$$

By (v), there exists $\rho_{n}>0$ such that

$$
F\left(x_{0}, y\right) \cap B\left[z_{0}, \delta\left(t_{n}\right)\right] \neq \emptyset, \quad \forall y \in B\left[y_{0}, \rho_{n}\right] .
$$

Do this for each $n$, and we can arrange that $\rho_{n} \downarrow 0$ as $n \rightarrow \infty$ and each $\rho_{n}<\rho$. Note that $B\left[z_{0}, \delta\left(t_{n}\right)\right] \subseteq V^{3 s}$ since $\delta\left(t_{n}\right)<s$ and $z_{0} \in V^{4 s}$. By (4.4) and (4.5) one then has for each $n \geq n_{0}$ that

$$
z_{0} \in B\left[F\left(x_{0}, y\right) \cap V^{3 s} ; \delta\left(t_{n}\right)\right] \subseteq F^{y}\left(B\left[x_{0}, T_{n}\right]\right)
$$

and so $z_{0} \in F^{y}\left(x^{n}(y)\right)$ for some $x^{n}(y) \in B\left[x_{0}, T_{n}\right]$. Do this for each $n \geq n_{0}$ and define $x\left(y_{0}\right)=x_{0}$ and $x(y)=x^{n}(y)$ for $y$ with $\rho_{n+1}<d\left(y, y_{0}\right) \leq \rho_{n}$. Then $x(y) \rightarrow x_{0}$ as $y \rightarrow y_{0}$ and so clearly $x(\cdot)$ is a function from $B\left[y_{0}, \rho_{n_{0}}\right]$ into $B\left[x_{0}, T_{n_{0}}\right]$ with the desired properties.

We end this paper with a simple example. Let $h$ be a discontinuous linear functional on a Banach space $X$ (say over $\mathbb{R}$ ); thus it is unbounded on any ball $B$ in $X$ and hence it maps $B$ onto $\mathbb{R}$. Let $g$ be any function from $\mathbb{R}$ onto itself, and $f:=g \circ h$; then $f$ also maps any ball $B$ onto $\mathbb{R}$ and so must be $(\delta(t), t)$-open with $Z=\mathbb{R}$, where $\delta$ can be any monotone function on $\mathbb{R}^{+}$. Let $Y=\mathbb{R}$ and $F: X \times Y \rightarrow \mathbb{R}$ be defined by

$$
F(x, y)=f(x)+\sqrt{x}+y .
$$

Then each $F^{y}$ is $\alpha$-approximated by $f$ (with $U=X, V=\mathbb{R}=V^{f}$ ) where $\alpha(t):=\sqrt{t}$, because of the elementary inequality

$$
\left|\sqrt{\left|x_{1}\right|}-\sqrt{\left|x_{2}\right|}\right| \leq \sqrt{\left|x_{1}-x_{2}\right|}, \quad \forall x_{1}, x_{2} \in X .
$$

Therefore, applying Theorem 4.2 with any $\delta$ such that $\alpha \prec \delta$, we see that $F$ is an open mapping; also one may apply Theorem 4.3 to conclude that there exists an implicit function for $z_{0} \in F(x, y)$. Similar examples of multifunctions can also be found. We note further that, our function $f$ is, in general not 1-1 and therefore not covered by the results in [21]. 


\section{Acknowledgements}

The authors gratefully acknowledge the financial supports from the Institute of Mathematical Sciences, Chinese University of Hong Kong and from the Research Grant Council of Hong Kong.

\section{References}

[1] J.-P. Aubin and H. Frankowska, Set valued analysis (Birkhäuser, Boston, 1990).

[2] D. Azé, 'An inversion theorem for set-valued maps', Bull. Austral. Math. Soc. 37 (1988), 411-414.

[3] D. Azé, C. C. Chou and J. P. Penot, 'Subtraction theorems and approximate openness for multifunctions: topological and infinitesimal view-points', preprint.

[4] J. M. Borwein, 'Stability and regular points of inequality systems', J. Optim. Theory Appl. 48 (1986), 9-52.

[5] J. M. Borwein and D. M. Zhang, 'Verifiable necessary and sufficient condition for openness and regularity of set-valued maps', J. Math. Anal. Appl. 134 (1988), 441-459.

[6] C. C. Chou, 'Sur quelques théorèmes d'inversion locale', C. R. Acad. Sci. Paris, Sér. I Math. 316 (1993), 19-22.

[7] S. Dolecki, 'Semicontinuity in constrained optimization, part I. 1. Metric spaces', Control Cybernet. 7 (1978), 5-15.

[8] - 'Semicontinuity in constrained optimization, part Ib. 1. Normed spaces', Control Cybernet. 7 (1978), 17-26.

[9] H. Frankowska, 'An open mapping principle for set-valued maps', J. Math. Anal. Appl. 127 (1987), 172-180.

[10] - 'Some inverse mapping theorems', Ann. Inst. H. Poincaré Anal. Non Linéaire 7 (1990), 183-234.

[11] L. A. Liusternik and V. J. Sobolev, Elements of functional analysis (Unger, New York, 1961).

[12] D. Luenberger, Optimization by vector spaces methods (Wiley, New York, 1968).

[13] K. F. Ng, 'An open mapping theorem', Math. Proc. Cambridge Philos. Soc. 74 (1973), 61-63.

[14] _ 'An inequality implicit function theorem', J. Austral. Math. Soc. (Series A) 44 (1988), $146-150$.

[15] J.-P. Penot, 'Inversion à droite d'applications non linearires et applications', C. R. Acad. Sci.Paris, Sér. I Math. 290 (1980), 997-1000.

[16] — 'Open mappings theorems and linearization stability', Numer. Funct. Anal. Optim. 8(1985), 21-35.

[17] _ 'Metric regulariy, openness and Lipschitzian behavior of multifunctions', Nonlinear Anal. 13 (1989), 629-643.

[18] S. M. Robinson, 'Normed convex processes', Trans. Amer. Math. Soc. 174 (1972), 127-140.

[19] - 'Regularity and stability for convex multivalued functions', Math. Oper. Res. 1 (1976), $130-143$.

[20] — 'Stability theory for systems of inequalitys. part II: differentiable non-linear systems', SIAM J. Numer. Anal. 13 (1976), 497-513.

[21] _ - 'An implicit function theorem for a class of nonsmooth functions', Math Oper. Res. 16 (1991), 292-309.

[22] C. Ursescu, 'Multifunctions with closed convex graphs', Czechoslovak Math. J. 25 (1975), 438449. 
[23] — 'Tangency and openness of multifunctions in Banach spaces', An. Ştünt. Univ. “Al. I. Cuza", Iasi Sect. I. a Mat. (N.S.) 34 (1988), 221-226.

[24] J. Zowe and S. Kureyusz, 'Regularity and stabiltiy for the mathematical programming problem in Banach spaces', Appl. Math. Optim. 5 (1979), 49-62.

Université de Perpignan

France
South China Normal University

Guangzhou

China

The Chinese University of Hong Kong

Hong Kong

e-mail: ngkf@cuhk.hk 